\title{
Clear cell chondrosarcoma in Von Hippel-Lindau disease
}

\author{
Koen M. A. Dreijerink ${ }^{1,2}$ (D) Rachel S. van Leeuwaarde ${ }^{3} \cdot$ Wenzel M. Hackeng $^{2} \cdot$ Rachel H. Giles $^{4}($ ) \\ Wendy W. J. de Leng ${ }^{2}$. Paul C. Jutte ${ }^{5}$ - Albert J. H. Suurmeijer ${ }^{6}$ (อ) Bernadette P. M. van Nesselrooij ${ }^{7}$. \\ Lodewijk A. A. Brosens ${ }^{2}$
}

Published online: 31 October 2019

(c) The Author(s) 2019

\begin{abstract}
A diagnosis of clear cell chondrosarcoma of the ulna was made in a patient with Von Hippel-Lindau disease (VHL). After surgery, genetic analysis of the tumor tissue showed loss of heterozygosity at the $V H L$ gene locus. Immunohistochemical analysis confirmed loss of expression of the VHL protein in the tumor cells. In addition, abundant Cyclin D1 expression in the tumor was observed. Chondrosarcoma has been described before in a VHL patient and VHL protein expression has been correlated to tumor grade in a series of sporadic chondrosarcomas. In this report, we show that clear cell chondrosarcoma may be a rare but canonical VHL manifestation through a cell-autonomous mechanism involving somatic loss-of-heterozygosity of the $V H L$ tumor suppressor gene. We discuss the relevance of this observation with regard to the pathogenesis of clear cell chondrosarcoma in the context of VHL.
\end{abstract}

Keywords Clear cell chondrosarcoma $\cdot$ Von Hippel-Lindau disease $\cdot V H L$ gene $\cdot$ Cyclin D1

\section{Introduction}

Von Hippel-Lindau disease (VHL) is an autosomal dominantly inherited tumor syndrome that is caused by heterozygous inactivating mutations of the $V H L$ tumor suppressor gene, usually in the germline [1]. Biallelic loss of $V H L$ gene

Koen M. A. Dreijerink

k.dreijerink@amsterdamumc.nl

1 Department of Internal Medicine, Amsterdam University Medical Centers, Location VU University Medical Center, De Boelelaan 1117, 1081 HV Amsterdam, The Netherlands

2 Department of Pathology, University Medical Center Utrecht, Utrecht, The Netherlands

3 Department of Endocrine Oncology, University Medical Center Utrecht, Utrecht, The Netherlands

4 Department of Nephrology and Hypertension, Regenerative Medicine Center, University Medical Center Utrecht, Utrecht, The Netherlands

5 Department of Orthopedics, University Medical Center Groningen, Groningen, The Netherlands

6 Department of Pathology, University Medical Center Groningen, Groningen, The Netherlands

7 Department of Medical Genetics, University Medical Center Utrecht, Utrecht, The Netherlands function through somatic loss of the second allele results in a state of pseudo hypoxia in cells that leads to angiogenesis and tumor or cyst formation [2]. VHL is characterized by tumors of the retina, brain and myelum (retinal and CNS hemangioblastomas), kidneys (clear cell renal cell carcinoma), adrenal glands (pheochromocytoma), endocrine pancreas (pancreatic neuroendocrine tumors) and endolymphatic sac and epididymis and broad ligament cystadenomas, in addition to potentially clinically relevant complex and simple cysts in the kidney, pancreas, adrenal gland, and associated with CNS hemangioblastomas (syrinx).

Chondrosarcomas are malignant tumors of the bone with variable morphology and clinical behavior that are characterized by the production of cartilage matrix. We report a case of clear cell chondrosarcoma in a VHL patient. Genetic analysis showed loss of heterozygosity (LOH) at the $V H L$ gene locus. Loss of expression of the VHL protein (pVHL) suggests that clear cell chondrosarcoma may be part of the VHL tumor spectrum. 


\section{Subject and methods}

At age 48, a female VHL patient presented with pain in her left arm. She was diagnosed with VHL at age 25. Genetic testing of peripheral blood had confirmed a c.500G $>$ A (p.Arg167Gln) VHL gene mutation. Her medical history included surgery for central nervous system hemangioblastomas at age 26, 28, 39 and 45 as well as a retinal hemangioma for which she underwent a coagulation procedure at age 26. Imaging studies suggested a lytic lesion in the left distal ulna (Fig. 1). A biopsy was taken that was suggestive of clear cell chondrosarcoma. Additional immunohistochemical analysis showed strong S-100 staining. Antibodies directed at cytokeratins 8, 18, AE1-3 and also inhibin, which are used for the identification of renal cell carcinoma or hemangioblastoma, were negative. Bone scintigraphy did not reveal any other bone lesions. Surgical resection resulted in complete removal of the tumor. Tumor tissue was frozen after surgery. DNA was isolated and after PCR amplification the VHL gene locus was analyzed by Sanger sequencing [3]. In addition, tumor DNA was subjected to comprehensive genetic and epigenetic analysis using an Ion Ampliseq Cancer Hotspot v2 panel (Thermo Fisher Scientific), Infinium CytoSNP-850 K and Infinium MethylationEPIC-850 K beadchip (Illumina). In parallel, tissue was processed for immunohistochemical analysis using monoclonal antibodies directed at pVHL and the pVHL-suppressed target Cyclin D1 (VHL, Cat.no. 556347, 1:200, BD Biosciences; Cyclin D1(SP4), Cat.no. 241R-15, 1:100, Cell Marque). The patient was informed and gave written permission for this case report.
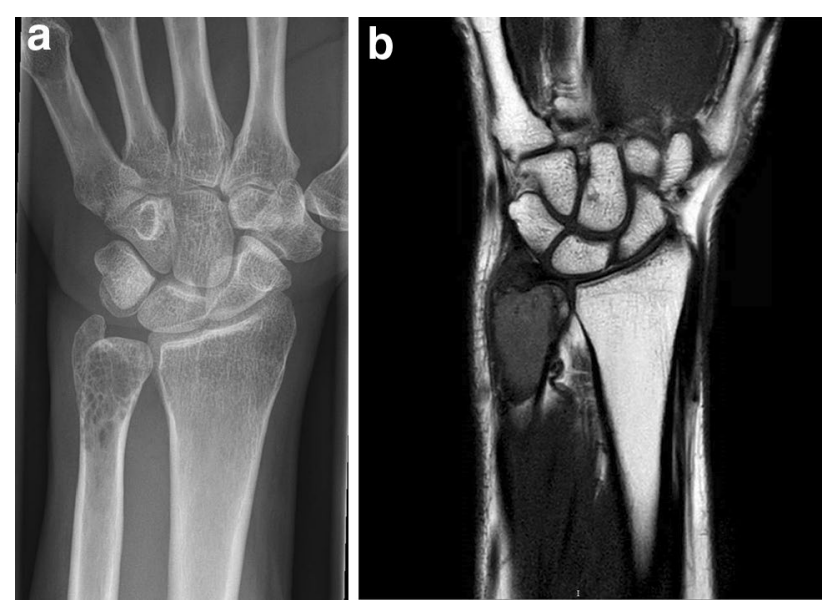

Fig. 1 Imaging of a clear cell chondrosarcoma in a VHL patient. a X-ray of the left forearm. The lesion is located in the distal ulna. b Magnetic resonance imaging of the lesion

\section{Results}

Histological examination showed a tumor composed of sheets of cells with clear to slightly eosinophilic vacuolated cytoplasm and central nuclei with small central nucleoli (Fig. 2a). Numerous osteoclast-type giant cells and reactive bone cells were present in between the tumor cells. The

a $\mathrm{HE}$

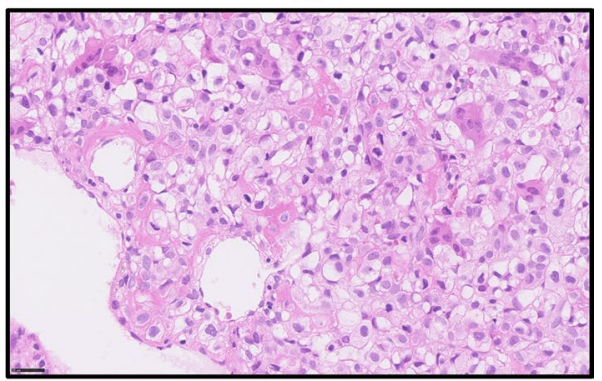

b $\quad \mathrm{pVHL}$
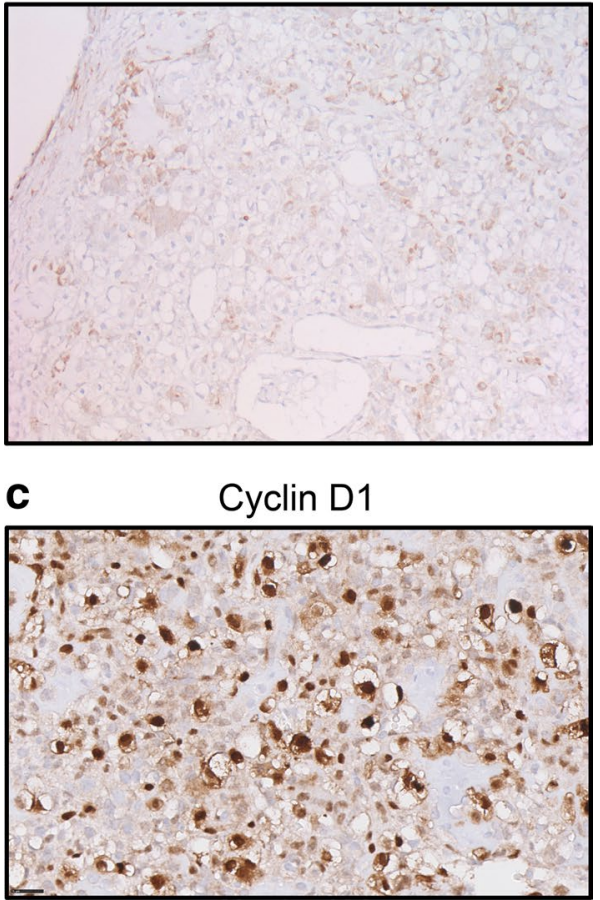

Fig. 2 Molecular analysis of the tumor after surgical removal of the clear cell chondrosarcoma. a Hematoxylin and eosin (HE) stain of the tumor tissue ( $\times 40$ magnification). The tumor is composed of cells with distinct borders and clear to slightly eosinophilic cytoplasm with central nuclei. Osteoclast-type giant cells, reactive bone and wide vessels are present in the tumor. $\mathbf{b}$ Immunohistochemical staining of the tumor tissue using antibodies directed at pVHL $(20 \times)$. Positive staining is observed in non-neoplastic multinucleated giant cells and other histiocytic cells present in the tumor, which are CD68 positive (not shown). c Immunohistochemical staining using antibodies directed at Cyclin D1 shows abundant expression in the tumor cells $(\times 40)$ 


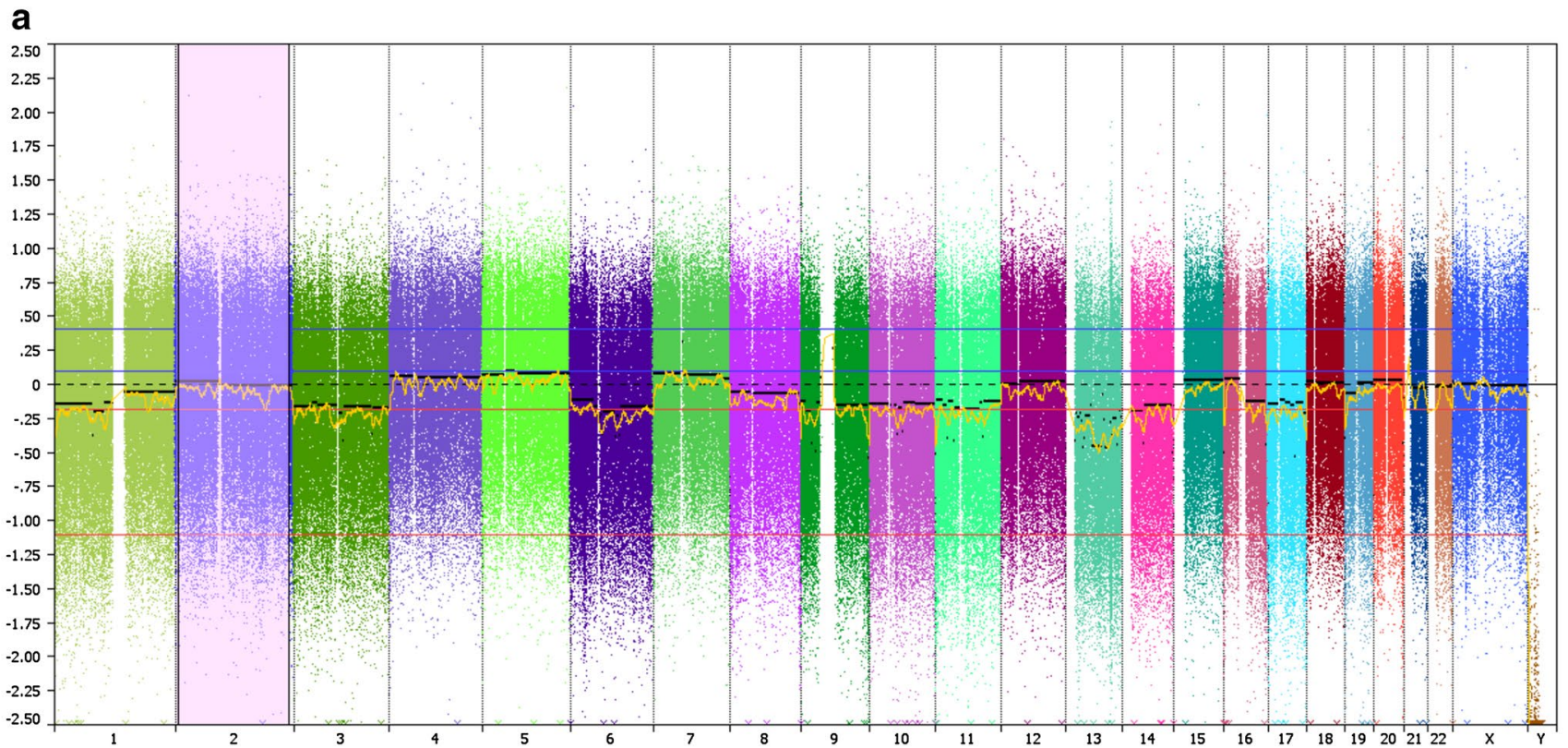

b

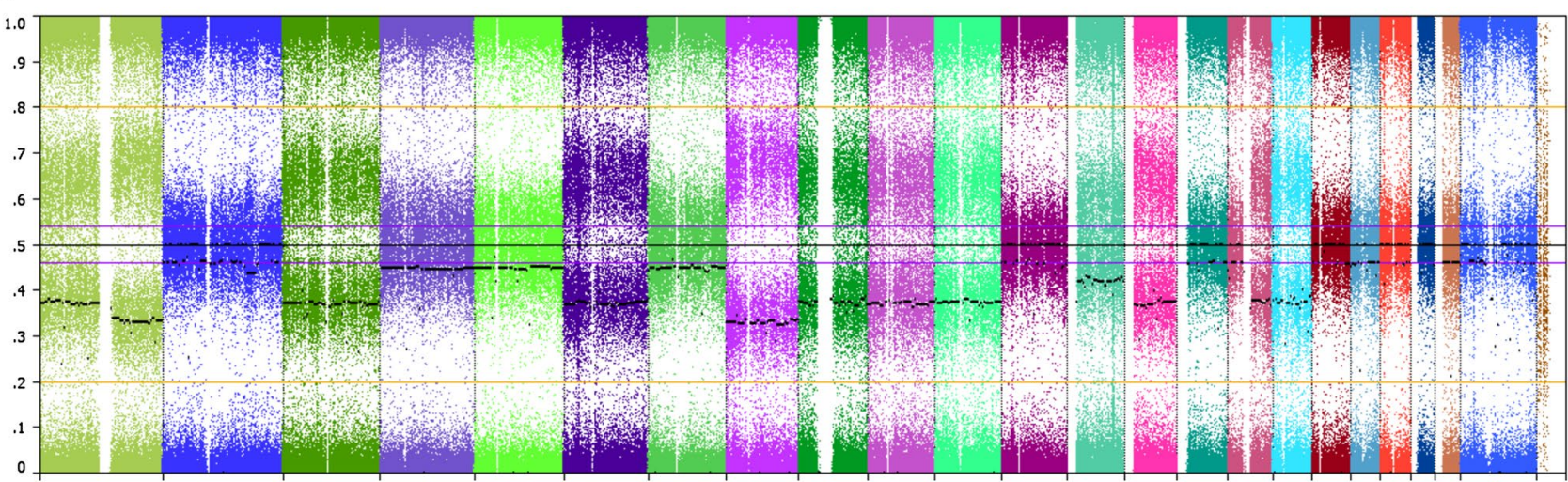

Fig. 3 SNP array indicating chromosomal gains and losses in the chondrosarcoma. a Copy number variations for each chromosome (log2 ratio). b B-allele frequencies

estimated tumor cell fraction of the micro dissected material was $80 \%$. Genetic analysis of the micro dissected tissue showed a higher chromatographic signal of the mutant allele than the wild type allele, suggestive for loss of the wild type allele (data not shown). Gene panel analysis confirmed the $V H L$ gene mutation in the tumor. No other mutations were found, except a non-pathogenic variant of the ataxia-telangiectasia mutated $(A T M)$ gene $(c .1810 \mathrm{C}>\mathrm{T}$; p.(Pro604Ser)). Allelic loss of $V H L$ was confirmed by SNP array (Fig. 3). Copy number variations included a monosomal pattern of chromosomes 3 (harboring the VHL gene), 6, 9 (CDKN2A), 10,11,13,14, and 17. Also loss of $1 \mathrm{p}$ and $16 \mathrm{q}$ was found and copy neutral LOH at 1q and 8 . The methylation array confirmed the above described copy number profile. No aberrant $\mathrm{CpG}$ methylation was observed at the $V H L$ promoter (data not shown). To determine the effect of the loss of the wild type $V H L$ allele on pVHL expression, we subsequently performed immunohistochemistry. Distinctly lower levels of pVHL were observed in the tumor cells as compared to adjacent non-neoplastic cells (Fig. 2b). These results indicate that a second genetic hit occurred in trans during tumor development that resulted in loss of pVHL. Profound expression of Cyclin D1 was observed in the tumor (Fig. 2c).

\section{Discussion}

We describe a case of a VHL patient with a primary clear cell chondrosarcoma. Loss of function of the wild type $V H L$ allele in the tumor and loss of $\mathrm{pVHL}$ expression suggest that the chondrosarcoma is a canonical VHL manifestation in 
this patient. Cyclin D1 could be the driver of cell proliferation in this tumor.

Approximately $75-90 \%$ of chondrosarcomas are so-called conventional chondrosarcomas (reviewed in [3]). These tumors rarely present in childhood or adolescence and most often are located in the tubular bones [4]. In conventional chondrosarcomas, mutations in the isocitrate dehydrogenase $(I D H)$ genes are the most prevalent genetic events, in up to $59 \%$ of cases in a comprehensive exome sequencing study [5]. In addition, TP53 gene function (20\%), the retinoblastoma pathway $(33 \%)$ and hedgehog signaling (18\%) are affected by somatic mutations in the tumors. Previously, a mildly differentiated chondrosarcoma has been reported in a VHL patient. However, no genetic or immunohistochemical analysis of the tumor was performed in this patient [6]. Chen et al. assessed VHL mRNA and protein levels in a series of conventional chondrosarcomas [7]. Levels of both $V H L$ mRNA and protein were found to be reduced in a significant proportion of chondrosarcomas compared with adjacent normal tissue. Low expression of pVHL was associated with apoptosis and tumor grade.

Clear cell chondrosarcoma is a rare subtype of non-conventional chondrosarcoma comprising approximately $2 \%$ of all chondrosarcoma cases and is considered a low-grade variant. It occurs predominantly at the epiphyseal ends of long bones. There are no known inherited causes for clear cell chondrosarcoma. Due to its rarity, limited information is available on the molecular background of this subtype. Meijer et al. reported that expression of the protein p16Ink4a, encoded by the $C D K N 2 A$ gene, was absent in 20 out of 21 sporadic clear cell chondrosarcoma cases [8]. P16 inhibits expression of the Cyclin D1 (CCND1) gene and high expression of $C C N D 1$ was observed in $42 \%$ of tumors tested. We also found $\mathrm{LOH}$ at the $C D K N 2 A$ gene locus and abundant Cyclin D1 expression in the tumor in this VHL patient. In collaboration with cyclin-dependent kinases (CDK) 4 and 6, Cyclin D1 stimulates progression through the G1 phase of the cell cycle via retinoblastoma protein inactivation, which may lead to tumor formation. CDK4/6 inhibitors represent a promising new cancer treatment option.

The clear cell histology in renal cell carcinoma is known to be a result of accumulation of cytoplasmic lipids and glycogen. Lipid accumulation has been described in other VHL-associated tumors, such as pancreatic neuroendocrine tumors [9]. Although speculative, it is interesting to consider that the role of $\mathrm{pVHL}$ in clear cell chondrosarcoma is analogous and initiates similar metabolic changes in tumor development. The VHL protein functions as part of a ubiquitin E3 ligase that targets the alpha-subunits of the hypoxia inducible factor (HIF) by inducing oxygen-dependent degradation through ubiquitylation. Accumulation of nuclear HIF in a cell that has lost pVHL function results in permanent transcription of hypoxia-induced genes [2]. Interestingly, the
CCND1 gene, which has been found up regulated in clear cell chondrosarcomas, has also been reported as a pVHLdependent HIF target gene in renal cell carcinoma [10]. Thus, the strong Cyclin D1 expression observed in the VHL tumor may be a direct result of the loss of pVHL-dependent transcriptional repression. Due to their pseudo hypoxic state, VHL-related tumors tend to be highly vascularized and anti-angiogenic compounds are routinely used for the treatment of advanced disease. Chondrosarcomas have also been successfully treated with anti-angiogenic drugs, further indicating common underlying mechanisms of development and treatment of these tumor types.

To conclude, we report a clear cell chondrosarcoma in a VHL patient that exhibited loss of pVHL expression due to loss of expression of the $V H L$ gene. Our data support the notion that clear cell chondrosarcoma could be a rare but specific manifestation of VHL. Increased Cyclin D1 function leading to vascularized clear cell tumors may be a common molecular feature of VHL-associated tumors and clear cell chondrosarcoma, which could be useful for therapeutic purposes in the future.

\section{Compliance with ethical standards}

Ethical approval All procedures performed in studies involving human participants were in accordance with the ethical standards of the institutional and/or national research committee and with the 1964 Helsinki declaration and its later amendments or comparable ethical standards.

Informed consent Informed consent was obtained from all individual participants included in the study.

Open Access This article is distributed under the terms of the Creative Commons Attribution 4.0 International License (http://creativeco mmons.org/licenses/by/4.0/), which permits unrestricted use, distribution, and reproduction in any medium, provided you give appropriate credit to the original author(s) and the source, provide a link to the Creative Commons license, and indicate if changes were made.

\section{References}

1. Latif F, Tory K, Gnarra J, Yao M, Duh FM, Orcutt ML, Stackhouse T, Kuzmin I, Modi W, Geil L et al (1993) Identification of the von Hippel-Lindau disease tumor suppressor gene. Science 260(5112):1317-1320

2. Kaelin WG Jr (2008) The von Hippel-Lindau tumour suppressor protein: O2 sensing and cancer. Nat Rev Cancer 8(11):865-873. https://doi.org/10.1038/nrc2502

3. Speetjens FM, de Jong Y, Gelderblom H, Bovee JV (2016) Molecular oncogenesis of chondrosarcoma: impact for targeted treatment. Curr Opin Oncol 28(4):314-322. https://doi.org/10.1097/ CCO.0000000000000300

4. Fletcher CDM, World Health Organization, International Agency for Research on Cancer (2013) WHO classification of tumours of 
soft tissue and bone. World Health Organization classification of tumours, 4th edn. IARC Press, Lyon

5. Tarpey PS, Behjati S, Cooke SL, Van Loo P, Wedge DC, Pillay N, Marshall J, O'Meara S, Davies H, Nik-Zainal S, Beare D, Butler A, Gamble J, Hardy C, Hinton J, Jia MM, Jayakumar A, Jones D, Latimer C, Maddison M, Martin S, McLaren S, Menzies A, Mudie L, Raine K, Teague JW, Tubio JM, Halai D, Tirabosco R, Amary F, Campbell PJ, Stratton MR, Flanagan AM, Futreal PA (2013) Frequent mutation of the major cartilage collagen gene COL2A1 in chondrosarcoma. Nat Genet 45(8):923-926. https:// doi.org/10.1038/ng.2668

6. Marinozzi A, Papapietro N, Barnaba SA, Di Martino A, Tonini G, Denaro V (2007) Chondrosarcoma of the iliac wing in Von Hippel-Lindau disease. J Exp Clin Cancer Res 26(4):599-601

7. Chen C, Zhou H, Liu X, Liu Z, Ma Q (2011) Reduced expression of von Hippel-Lindau protein correlates with decreased apoptosis and high chondrosarcoma grade. J Bone Joint Surg Am 93(19):1833-1840. https://doi.org/10.2106/JBJS.I.01553

8. Meijer D, de Jong D, Pansuriya TC, van den Akker BE, Picci P, Szuhai K, Bovee JV (2012) Genetic characterization of mesenchymal, clear cell, and dedifferentiated chondrosarcoma. Genes
Chromosom Cancer 51(10):899-909. https://doi.org/10.1002/ gcc. 21974

9. Lubensky IA, Pack S, Ault D, Vortmeyer AO, Libutti SK, Choyke PL, Walther MM, Linehan WM, Zhuang Z (1998) Multiple neuroendocrine tumors of the pancreas in von Hippel-Lindau disease patients: histopathological and molecular genetic analysis. Am J Pathol 153(1):223-231. https://doi.org/10.1016/S0002 -9440(10)65563-0

10. Raval RR, Lau KW, Tran MG, Sowter HM, Mandriota SJ, Li JL, Pugh CW, Maxwell PH, Harris AL, Ratcliffe PJ (2005) Contrasting properties of hypoxia-inducible factor 1 (HIF-1) and HIF-2 in von Hippel-Lindau-associated renal cell carcinoma. Mol Cell Biol 25(13):5675-5686. https://doi.org/10.1128/ MCB.25.13.5675-5686.2005

Publisher's Note Springer Nature remains neutral with regard to jurisdictional claims in published maps and institutional affiliations. 